\title{
OWEN'S MODEL OF A GENETICAL SYSTEM WITH DIFFERENTIAL VIABILITY BETWEEN SEXES
}

\author{
S. P. H. MANDEL \\ Mathematics-Statistics Unit, Division of Research in Epidemiology and Communications Science, \\ World Health Organization, Geneva, Switzerland
}

Received 16.ii.70

\section{INTRODUCTION}

Following the derivation by Haldane $(1924,1926)$ of the basic equations describing the changes in gene frequency with random mating for various methods of selection, Owen $(1952,1953)$ developed the idea of a genetical system in which the genotypic viabilities differ between females and males as well as between the genotypes themselves. In particular, he showed that, in this situation, it is possible for two distinct stable equilibrium states to exist. Some special cases of such a system have been investigated and discussed by Li (1963, 1967), and Bodmer (1965) has analysed the situation in considerable detail; however, apart from these contributions, Owen's interesting model has been given relatively little attention. It is the purpose of this paper to examine, in a general way and on the basis of the equations obtained by Owen, some of the properties of such a system.

\section{The mathematical Model}

First we summarise the mathematical equations, derived by Owen, which describe the system. With some slight modifications of Owen's notation, we consider a pair of alleles $A, a$ at a single genetic locus in a random mating diploid population. Let the relative viabilities of the three genotypes $A A, A a$ and $a a$ be denoted respectively by the non-negative numbers $a_{i}, h_{i}$ and $b_{i}$ where $i=1$ corresponds to females and $i=2$ to males. Thus the model is specified by these six viability parameters. Furthermore, let $p_{1}$ and $p_{2}$ be the gene frequencies of the allele $A$ in females and males respectively in a specified generation. Then it is shown in Owen (1953) that, assuming random mating, the relationships between the gene ratios

$$
u_{i}=\frac{p_{i}}{1-p_{i}}, \quad(i=1,2)
$$

in successive generations are given by the equations

$$
u_{i}^{\prime}=\frac{2 a_{i} u_{1} u_{2}+h_{i}\left(u_{1}+u_{2}\right)}{h_{i}\left(u_{1}+u_{2}\right)+2 b_{i}}, \quad(i=1,2)
$$

The pair of recurrence relations (2) describe the dynamics of the system.

At equilibrium $u_{1}^{\prime}=u_{1}, u_{2}^{\prime}=u_{2}$ and setting $u_{2}=u_{1} t$ the values of the ratio $u_{1}$ are given by the equations

$$
u_{1}=\frac{t-c_{1}}{1-C_{1} t}, \quad u_{1}=\frac{c_{2} t-1}{C_{2} t-t^{2}}
$$

D 
with

$$
\left.\begin{array}{rl}
c_{1} & =\frac{2 b_{1}}{h_{1}}-1, c_{2}=\frac{2 b_{2}}{h_{2}}-1 \\
C_{1} & =\frac{2 a_{1}}{h_{1}}-1, C_{2}=\frac{2 a_{2}}{h_{2}}-1
\end{array}\right\}
$$

and where $t$ satisfies the cubic equation

with

$$
\begin{gathered}
f(t)=t^{3}-3 \alpha t^{2}+3 \beta t-1=0 \\
3 \alpha=C_{1} c_{2}+c_{1}+C_{2}, 3 \beta=C_{2} c_{1}+c_{2}+C_{1} .
\end{gathered}
$$

Considering the question of stability, we note that the basic equations (2) can be written in the form

$$
u_{1}^{\prime}=\Phi_{1}\left(u_{1}, u_{2}\right), u_{2}^{\prime}=\Phi_{2}\left(u_{1}, u_{2}\right)
$$

and considering small displacements $x_{1}, x_{2}$ in the values of $u_{1}, u_{2}$ giving rise to corresponding changes $x_{1}^{\prime}, x_{2}^{\prime}$ in $u_{1}^{\prime}, u_{2}^{\prime}$, we have

$$
\begin{aligned}
u_{1}^{\prime}+x_{1}^{\prime} & =\Phi_{1}\left(u_{1}+x_{1}, u_{2}+x_{2}\right) \\
& =\Phi_{1}\left(u_{1}, u_{2}\right)+x_{1} \frac{\partial \Phi_{1}}{\partial u_{1}}+x_{2} \frac{\partial \Phi_{1}}{\partial u_{2}}
\end{aligned}
$$

approximately.

For displacements near the equilibrium, we have then

$$
x_{1}^{\prime}=b_{11} x_{1}+b_{12} x_{2}, x_{2}^{\prime}=b_{21} x_{1}+b_{22} x_{2}
$$

where

$$
\begin{aligned}
b_{i j} & =\frac{\partial \Phi_{i}}{\partial u_{f}} \\
& =\frac{2 a_{i}\left(u_{1}+u_{2}\right)+h_{i}\left(1-u_{i}\right)-2 a_{i} u_{j}}{h_{i}\left(u_{1}+u_{2}\right)+2 b_{i}}
\end{aligned}
$$

(using (2), and substituting the equilibrium values of $u_{1}, u_{2}$ after differentiating) for $i, j=1,2$.

The difference equations (9) have solutions of the form

$$
x_{1}^{(n)}=A \lambda_{1}^{n}+B \lambda_{2}^{n}, x_{2}^{(n)}=C \lambda_{1}^{n}+D \lambda_{2}^{(n)}
$$

where $A, B, C$ and $D$ are determined by the $\left(b_{i j}\right)$ and by the initial values of the displacements $x_{1}$ and $x_{2}$, and $\lambda_{1}$ and $\lambda_{2}$ are the latent roots of the matrix $B=\left(b_{i j}\right)$. Thus the question of stability near the equilibrium is determined by examining the absolute magnitudes of $\lambda_{1}$ and $\lambda_{2}$, which are thus sometimes called the stability roots of the equilibrium state.

\section{The initial PROgRess of NeW genes}

Necessary and sufficient conditions for a new gene, arising in a population by migration or mutation, to increase in frequency and become established in that population have been derived and discussed for a variety of genetical systems by different writers (Bodmer and Parsons, 1960; Parsons, $1961 a, b$; Parsons, 1962; Mandel, 1963; Bodmer, 1965). 
Suppose that the allele $A$ is introduced with low frequency into a population consisting initially only of genes $a$. This implies that we are considering the stability of the equilibrium state represented by $u_{1}=u_{2}=0$, so that we have to solve the pair of difference equations (9) with

$$
b_{i j}=\frac{h_{i}}{2 b_{i}}, \quad(i, j=1,2)
$$

The latent roots of the matrix $\left(b_{i j}\right)$ in this case are clearly $\frac{1}{2}\left\{\frac{h_{1}}{b_{1}}+\frac{h_{2}}{b_{2}}\right\}$ and zero, so that the solutions are

$$
\begin{aligned}
& x_{1}^{(n)}=\frac{h_{1}}{2^{n} b_{1}}\left\{\frac{h_{1}}{b_{1}}+\frac{h_{2}}{b_{2}}\right\}^{n-1}\left(x_{1}+x_{2}\right) \\
& x_{2}^{(n)}=\frac{h_{2}}{2^{n} b_{2}}\left\{\frac{h_{1}}{b_{1}}+\frac{h_{2}}{b_{2}}\right\}^{n-1}\left(x_{1}+x_{2}\right)
\end{aligned}
$$

from which it follows immediately that the necessary and sufficient condition for the frequency of the newly introduced allele to increase is

$$
\frac{h_{1}}{b_{1}}+\frac{h_{2}}{b_{2}}>2
$$

which is the result found by Parsons (1961a) and Bodmer (1965) on the basis of somewhat different methods of approach.

In the situation in which there is no difference in viabilities of the same genotype between sexes, which was first discussed by Fisher (1922), the condition (15) reduces to $h>b$ which is precisely the condition obtained by Bodmer and Parsons (1960). It might be noted that, in this model, a selective advantage in both sexes of the newly formed heterozygotes over the entrenched wild homozygotes is not a necessary condition for the invading allele to become established in the population. However, as Parsons (1961a) has observed, it is necessary that a selective disadvantage of the heterozygote in one sex must be balanced by a corresponding selective advantage of the heterozygote in the other sex.

\section{Lethal homozygotes}

Owen suggests that in the case where one allele $A$ is lethal in both sexes when in the homozygous condition $A A$ there are at most two non-trivial equilibria of which only one is stable. However, analysis of the situation reveals the following. If $A A$ is lethal in both sexes, we would have $a_{1}=a_{2}=0$, which implies

$$
C_{1}=C_{2}=-1
$$

and

$$
3 \alpha=c_{1}-c_{2}-1, \quad 3 \beta=c_{2}-c_{1}-1
$$

and hence the cubic equation (5) reduces to

$$
f(t)=(t+1)\left\{t^{2}-\left(c_{1}-c_{2}\right) t-1\right\}=0
$$


which has roots

$$
t=-1, \quad \frac{c_{1}-c_{2}-\sqrt{\left(c_{1}-c_{2}\right)^{2}+4}}{2}, \frac{c_{1}-c_{2}+\sqrt{\left(c_{1}-c_{2}\right)^{2}+4}}{2}
$$

only the last of which is positive and could therefore potentially lead to a non-trivial equilibrium state of the system. Thus when homozygotes of one kind are lethal in both sexes, there exists at most one non-trivial equilibrium state, and we proceed to derive the conditions under which it does exist, and to show that when it exists it is stable.

In order for the equilibrium to exist, we must have, from equation (3), the condition that

$$
u_{1}=\frac{t-c_{1}}{1+t}>0
$$

which is

$$
c_{1}-c_{2}+\sqrt{\left(c_{1}-c_{2}\right)^{2}+4}>2 c_{1}
$$

and this reduces to the condition

$$
c_{1} c_{2}<1
$$

(except when $c_{1},=c_{2}=-1$, recalling that $c_{1}, c_{2} \geqq-1$ ) which is equivalent to the condition

$$
\frac{h_{1}}{b_{1}}+\frac{h_{2}}{\bar{b}_{2}}>2
$$

and the latter in turn can be shown to be equivalent to the stability conditions found by Bodmer (1965).

It can also be shown that the equilibrium, when it exists, is always stable. For, in equations (9) and (10) we have

$$
\begin{aligned}
& b_{11}=b_{12}=\frac{h_{1}\left(1-u_{1}\right)}{h_{1}\left(u_{1}+u_{2}\right)+2 b_{1}} \\
& b_{21}=b_{22}=\frac{h_{2}\left(1-u_{2}\right)}{h_{2}\left(u_{1}+u_{2}\right)+2 b_{2}}
\end{aligned}
$$

so that the stability roots are

$$
\left\{\frac{h_{1}\left(1-u_{1}\right)}{h_{1}\left(u_{1}+u_{2}\right)+2 b_{1}}+\frac{h_{2}\left(1-u_{2}\right)}{h_{2}\left(u_{1}+u_{2}\right)+2 b_{2}}\right\} \text { and zero. }
$$

Examining the non-zero stability root, it can be written

$$
\begin{aligned}
\frac{1-u_{1}}{\left(u_{1}+u_{2}\right)+1+c_{1}}+\frac{1-u_{2}}{\left(u_{1}+u_{2}\right)+1+c_{2}} & =\frac{1-u_{1}}{1+t}+\frac{1-u_{2}}{(1+t)+\left(c_{2}-c_{1}\right)} \\
& =1-\frac{u_{1}\left(1+t^{2}\right)}{1+t}
\end{aligned}
$$

using equations (18) and (20) and recalling that $u_{2}=u_{1} t$.

Moreover, we note from (3) that

$$
1-u_{1}=1-\frac{t-c_{1}}{1+t}=\frac{1+c_{1}}{1+t}
$$


and

$$
1-u_{2}=1-\frac{1-c_{2} t}{1+t}=\frac{\left(1+c_{2}\right) t}{1+t}
$$

and hence

$$
\begin{aligned}
1-\frac{u_{1}\left(1+t^{2}\right)}{1+t} & =\frac{\left(1-u_{1}\right)+t\left(1-u_{2}\right)}{1+t} \\
& =\frac{\left(1+c_{1}\right)+\left(1+c_{2}\right) t^{2}}{(1+t)^{2}} \\
& \geqq 0
\end{aligned}
$$

with the inequality being strict unless $b_{1}=b_{2}=0$. Thus we have shown that the non-zero stability root lies between zero and 1, and that therefore the equilibrium, when it exists, is stable.

By way of illustration, we will show that the results obtained by $\mathrm{Li}$ (1963) follow very quickly from the equations derived above. Thus in Li's example (designated in his paper as Case I) in which there is selection against the viable homozygote in one sex (females) but not in the other, we would have

$$
c_{1}=1-2 s, \quad c_{2}=1, \quad(0<s \leqq 1)
$$

so that the single positive root of (18) would be

$$
t=-s+\sqrt{1+s^{2}}
$$

from which it follows that

$$
\begin{aligned}
u_{1}=\frac{t-c_{1}}{1+t} & =\frac{-s+\sqrt{1+s^{2}}-(1-2 s)}{1-s+\sqrt{1+s^{2}}} \\
& =\frac{s-1+\sqrt{1+s^{2}}}{1-s+\sqrt{1+s^{2}}}>0
\end{aligned}
$$

and this leads immediately to

$$
p_{1}=\frac{1}{2}-\frac{(1-s)}{2 \sqrt{1+s^{2}}}
$$

which is equivalent to equation (6) of $\mathrm{Li}$ (1963).

Moreover,

$$
\begin{aligned}
u_{2}=t u_{1} & =\left\{\sqrt{1+s^{2}}-s\right\} \frac{\sqrt{1+s^{2}}-(1-s)}{\sqrt{1+s^{2}}+(1-s)} \\
& =\frac{(1+s)-\sqrt{1+s^{2}}}{(1-s)+\sqrt{1+s^{2}}}>0
\end{aligned}
$$

and this leads directly to

$$
p_{2}=\frac{1}{2}\left\{1+s-\sqrt{1+s^{2}}\right\}
$$

which is equivalent to equations (5) of $\mathrm{Li}$ (1963).

D 2 
Next we consider Case II of $\mathrm{Li}$ (1963) in which there is differential selection against the viable homozygote in both sexes. This leads to the conditions

$$
c_{1}=1-2 s_{1}, \quad c_{2}=1-2 s_{2}, \quad\left(0<s_{1} \leqq 1,0<s_{2} \leqq 1\right)
$$

so that the single positive root of (18) would be

$$
t=\left(s_{2}-s_{1}\right)+\sqrt{1+\left(s_{2}-s_{1}\right)^{2}}
$$

from which it follows that

$$
u_{1}=\frac{\left(s_{1}+s_{2}-1\right)+\sqrt{1+\left(s_{1}-s_{2}\right)^{2}}}{\left(1-s_{1}+s_{2}\right)+\sqrt{1+\left(s_{1}-s_{2}\right)^{2}}}>0
$$

and, after a little algebra, that

$$
u_{1}=\frac{s_{1}\left(s_{1}-s_{2}\right)+\left(1-s_{1}\right)\left\{1-\sqrt{1+\left(s_{1}-s_{2}\right)^{2}}\right\}}{s_{1}-s_{2}}
$$

and it can readily be verified by means of some algebra that the latter is identical with equation (13) of $\mathrm{Li}$ (1963).

The numerical example $\left(s_{1}=0.325, s_{2}=0 \cdot 10\right)$ given by $\mathrm{Li}(1963)$ can be readily verified by substituting these numerical values in equation (39), which leads at once to $u_{1}=0 \cdot 25$.

\section{UnGonditionAL ADVANTAGE OF ONE GENE}

In the classical model without differential viability between sexes, it is well known that if one gene has an unconditional selective advantage over its allele then the gene ratio changes monotonically from one generation to the next until ultimately the inferior allele is eliminated altogether. Thus, dropping the suffixes temporarily to consider this case, we would have

$$
u^{\prime}=u \cdot \frac{a u+h}{h u+b}
$$

and if say $a<h<b$ then clearly

$$
a u+h<h u+b
$$

and

$$
u^{\prime}<u
$$

and hence no equilibrium state can exist.

In Owen's model with differential viability between sexes when one gene has an unconditional selective advantage over its allele in both sexes, we can show that the system has the following properties:

(i) The sum of the gene ratios changes monotonically from one generation to the next.

(ii) The gene ratio in both sexes is bounded by the average value of the gene ratios in the previous generation.

(iii) Changes in the gene ratios in the individual sexes are not necessarily monotonic.

(iv) No equilibrium state can exist (Bodmer, 1965). 
Thus, if we consider the basic equations (2) with $a_{\ell}<h_{\imath}<b_{i}$ in both sexes, then

$$
u_{1}<\Psi(u), \quad u_{2}^{\prime}<\Psi(u)
$$

where

$$
\Psi(u)=\frac{2 u_{1} u_{2}+u_{1}+u_{2}}{u_{1}+u_{2}+2}
$$

and $\Psi(u)$ can be written in the form

$$
2 \Psi(u)=u_{1}+u_{2}-\frac{\left(u_{1}-u_{2}\right)^{2}}{u_{1}+u_{2}+2}
$$

whence

$$
u_{1}^{\prime}<\frac{1}{2}\left(u_{1}+u_{2}\right), u_{2}^{\prime}<\frac{1}{2}\left(u_{1}+u_{2}\right)
$$

which establishes properties (i) and (ii) above.

Property (iii) can readily be demonstrated by taking $u_{1}=0$. For we would thus have

$$
u_{1}^{\prime}=\frac{h_{1} u_{2}}{h_{1} u_{2}+2 b_{1}}
$$

so that

$$
0<u_{1}^{\prime}<\frac{1}{2} u_{2}
$$

Finally, property (iv) follows as an immediate corollary of property (i); or else it can be adduced directly from equations (3) and (4) by observing that, under the conditions given,

$$
c_{1}, c_{2}>1, C_{1}, C_{2}<1
$$

and hence no equilibrium is possible since clearly

$$
u_{1}=\frac{t-c_{1}}{1-C_{1} t}<0 \text { whenever } t \leqq 1
$$

and

$$
u_{1}=\frac{c_{2} t-1}{\mathrm{C}_{2} t-t^{2}}<0 \text { whenever } t \geqq 1 \text {. }
$$

6. SUFFICIENT CONDITIONS FOR THE EXISTENGE OF NON-TRIVIAL EQUILIBRIA

In this section we will show that if

and

$$
\left.\begin{array}{l}
\frac{h_{1}}{a_{1}}+\frac{h_{2}}{a_{2}}>2 \\
\frac{h_{1}}{b_{1}}+\frac{h_{2}}{b_{2}}>2
\end{array}\right\}
$$


or if

and

$$
\left.\begin{array}{l}
\frac{h_{1}}{a_{1}}+\frac{h_{2}}{a_{2}}<2 \\
\frac{h_{1}}{b_{1}}+\frac{h_{2}}{b_{2}}<2
\end{array}\right\}
$$

then there exists at least one non-trivial state of equilibrium.

For this it is convenient to note that, in the notation of equation (5),

$$
f\left(c_{1}\right)=\left(c_{1} c_{2}-1\right)\left(1-c_{1} C_{1}\right)
$$

and that

$$
f\left(\frac{1}{C_{1}}\right)=\frac{1}{C_{1}^{3}}\left(1-C_{1} C_{2}\right)\left(1-c_{1} C_{1}\right)
$$

so that

$$
C_{1}^{3} f\left(\frac{1}{C_{1}}\right)=\frac{1-C_{1} C_{2}}{c_{1} c_{2}-1} f\left(c_{1}\right) .
$$

Taking the pair of inequalities (52), the second member of this pair can be written

$$
\frac{1}{1+c_{1}}+\frac{1}{1+c_{2}}>1
$$

which implies either

$$
c_{1} c_{2}<1
$$

or else

$$
\left(1+c_{1}\right)\left(1+c_{2}\right)=0
$$

and the latter reduces to

$$
c_{1} c_{2} \leqq 1
$$

with equality only when $c_{1}=c_{2}=-1$, in view of the conditions

$$
1+c_{1} \geqq 0,1+c_{2} \geqq 0 \text {. }
$$

Thus it follows that the conditions (52) together imply

$$
\left.\begin{array}{r}
c_{1} c_{2} \leqq 1 \\
C_{1} C_{2} \leqq 1
\end{array}\right\}
$$

the equality in either case holding only when $c_{1}=c_{2}=-1$ or $C_{1}=C_{2}=-1$.

We can now identify four distinct cases as follows:

(i) $c_{1}>0, C_{1}>0$.

This implies that (62) holds with strict inequality, and hence, when $c_{1} C_{1} \neq 1$, it follows from (54) that $f\left(c_{1}\right) \neq 0$ and from (56) that $f\left(c_{1}\right)$ and $f\left(\frac{1}{C_{1}}\right)$ have opposite signs and hence $f(t)=0$ has at least one root inside the 
interval whose boundaries are $c_{1}$ and $\frac{1}{C_{1}}$. But $u_{1}=\frac{t-c_{1}}{1-C_{1} t}$ is positive everywhere inside this interval, and hence there is a non-trivial equilibrium.

If $c_{1} C_{1}=1$ we have

$$
f\left(c_{1}\right)=f\left(\frac{1}{C_{1}}\right)=0
$$

and

$$
u_{1}=\frac{t-c_{1}}{1-C_{1} t}
$$

is indeterminate at $t=c_{1}$. However,

$$
u_{1}=\frac{c_{2} t-1}{C_{2} t-t^{2}}=\frac{c_{1} c_{2}-1}{c_{1}\left(C_{2}-c_{1}\right)} \text { at } t=c_{1}
$$

and $u_{1}>0$ because $C_{2}<\frac{1}{C_{1}}=c_{1}$ and hence both the numerator and the denominator are negative. Thus again in this case there is a non-trivial equilibrium; moreover, it is interesting to note that it is unique since

$$
u_{1}=\frac{t-c_{1}}{1-C_{1} t}=-c_{1}<0
$$

for all values of $t$ except $t=c_{1}$.

(ii) $c_{1}>0, C_{1} \leqq 0$.

In this case $f\left(c_{1}\right)<0$ and hence $f(t)=0$ has at least one root $t>c_{1}$ and $u_{1}>0$ for all $t>c_{1}$.

(iii) $c_{1} \leqq 0, C_{1}>0$.

In this case it follows from (55) that $f\left(\frac{1}{C_{1}}\right)>0$ and hence that $f(t)=0$ has at least one root in the interval $0<t<\frac{1}{C_{1}}$; and $u_{1}>0$ everywhere in this interval.

(iv) $c_{1} \leqq 0, C_{1} \leqq 0$.

Clearly under these conditions

$$
u_{1}=\frac{t-c_{1}}{1-C_{1} t}>0
$$

for all positive values of $t$; and since $f(t)=0$ must have at least one positive root it follows that there exists at least one non-trivial equilibrium.

Similarly, it follows that conditions (53) imply

$$
c_{1} c_{2}>1, C_{1} C_{2}>1
$$

and hence $c_{1}$ and $C_{1}$ must both be positive and the same argument goes through as in (i) above. 
We have thus proved that either of (52) or (53) are sufficient conditions for the existence of at least one non-trivial equilibrium.

\section{MONOTONIG APPROAGH NEAR EQUILIBRIUM}

Some time ago Edwards (1967) raised the question of the monotonic convergence of the gene frequency and Mandel (1968) showed that in the classical model in which there are no viability differentials between sexes, the change in gene frequency (or, what amounts to the same thing, the gene ratio) is always in the same direction.

In Owen's model, we can show that the gene ratios are changing monotonically when the population is near an equilibrium state. Beginning with the set of equations (10) we have, for example,

$$
b_{11}=\frac{2 a_{1} u_{2}+h_{1}\left(1-u_{1}\right)}{h_{1}\left(u_{1}+u_{2}\right)+2 b_{1}}=\frac{\left(1+C_{1}\right) u_{2}+1-u_{1}}{u_{1}+u_{2}+\left(1+c_{1}\right)}
$$

The denominator in expression (64) is clearly positive. We now consider the numerator and set

whence

$$
\begin{aligned}
R & =\left(1+C_{1}\right) u_{2}+1-u_{1} \\
& =u_{1} t+u_{1}-t+c_{1}+1-u_{1} \text { (using (3)) } \\
& =\left(1+c_{1}\right)+t\left(u_{1}-1\right) \\
R & =\frac{\left(1+c_{1}\right)+\left(1+C_{1}\right) t u_{2}}{1+t} \geqq 0 .
\end{aligned}
$$

In fact, it can be shown that, for all $i, j=1,2$

$$
b_{i j}=\frac{1}{u_{1}+u_{2}} \cdot \frac{\left(1+c_{i}\right) u_{i}+\left(1+C_{i}\right)\left(u_{1}^{2}+u_{2}^{2}-u_{j}^{2}\right)}{\left(1+c_{i}\right)+\left(u_{1}+u_{2}\right)} \geqq 0 .
$$

Thus we have shown that the elements of the $2 \times 2$ matrix $\left(b_{i j}\right)$ are nonnegative and hence the latent roots $\lambda_{1}$ and $\lambda_{2}$ are real and the dominant latent root is positive. These properties follow from a theorem of Frobenius (1912) on the latent roots of matrices with non-negative elements. They can also be derived very easily by observing that the characteristic equation of the matrix $\left(b_{i j}\right)$ is

$$
\lambda^{2}-\left(b_{11}+b_{22}\right) \lambda+\left(b_{11} b_{22}-b_{12} b_{21}\right)=0
$$

whose discriminant is

$$
\begin{aligned}
\Delta & =\left(b_{11}+b_{22}\right)^{2}-4\left(b_{11} b_{22}-b_{12} b_{21}\right) \\
& =\left(b_{11}-b_{22}\right)^{2}+4 b_{12} b_{21}
\end{aligned}
$$

which is clearly non-negative so that the roots are real. Moreover

$$
\lambda_{1}+\lambda_{2}=b_{11}+b_{22} \geqq 0
$$

with equality only when $b_{11}=b_{22}=0$, which can occur only when both homozygotes in both sexes are lethal; in this case only heterozygotes of either sex would be present in the population and changes in gene frequency are therefore zero. Otherwise the dominant latent root is positive and it follows that oscillations of the gene ratio do not occur when the population 
is sufficiently close to an equilibrium state, that is to say changes in the gene ratio are monotonic.

\section{StABle EQUilibrium in THE ABSENCE OF HeTERosis}

It is easy to show, by means of a numerical example, that there exist stable equilibria in the absence of heterosis in either sex.

Thus if we take the situation in which, for example, the viability parameters are

we have

$$
\left.\begin{array}{lll}
a_{1}=1, & h_{1}=2, & b_{1}=3 \\
a_{2}=3, & h_{2}=2, & b_{2}=1
\end{array}\right\}
$$

and hence

$$
\left.\begin{array}{ll}
c_{1}=2, & C_{1}=0 \\
c_{2}=0, & C_{2}=2
\end{array}\right\}
$$

$$
\alpha=\beta=\frac{4}{3}
$$

so that equation (5) reduces to

$$
f(t)=(t-1)\left(t^{2}-3 t+1\right)=0
$$

whose roots are $0 \cdot 382,1$ and $2 \cdot 618$.

When $t=0.382, u_{1}=-1.618$; when $t=1, u_{1}=-1$. Hence both of these roots of (74) can be ignored. However, when $t=2 \cdot 618, u_{1}=0.618$ and $u_{2}=t u_{1}=1.618$ and hence the population is in equilibrium when the gene ratios have these values. In order to determine the stability, we can calculate the $b_{i j}$ from equations (67) and find that the matrix is

$$
B=\left(\begin{array}{ll}
0.382 & 0 \cdot 191 \\
1.309 & 0.382
\end{array}\right)
$$

so that the characteristic equation of $B$ is

$$
\lambda^{2}-0 \cdot 764 \lambda-0 \cdot 104=0
$$

and the stability roots are therefore 0.882 and $-0 \cdot 118$. The equilibrium is therefore stable.

This is interesting, as already remarked, because the heterozygote does not have a selective advantage over both homozygotes in either females or males. Moreover, it is interesting to note that, apart from the disparity between the equilibrium gene ratios in the two sexes, the population dynamics of this system are identical with those of a system in which there are no viability differences between sexes and in which the viability parameters have the values $a=3, \mathrm{~h}=4, b=3$.

\section{Two STABLE POLYMORPHIC EQUILIBRIA}

We apply the results of the previous sections to the examples given by Owen (1953) of genetical systems with two distinct stable equilibria in which both alleles are present in the population. 
It will be noted along the way that there appear to be one or two errors in the presentation of Owen's results; these are merely computational, and not errors in principle, and are quickly rectified.

In Owen's first example, the six viability parameters have the values

so that

$$
\left.\begin{array}{lll}
a_{1}=0 \cdot 5, & h_{1}=1, & b_{1}=0 \cdot 5 \\
a_{2}=2 \cdot 15, & h_{2}=1, & b_{2}=2 \cdot 15
\end{array}\right\}
$$

$$
\left.\begin{array}{l}
c_{1}=C_{1}=0 \\
c_{2}=C_{2}=3 \cdot 3
\end{array}\right\}
$$

so that the cubic equation (5) reduces to

$$
f(t)=(t-1)\left(t^{2}-2 \cdot 3 t+1\right)=0
$$

with roots $0 \cdot 5821,1$ and $1 \cdot 7179$.

Since, from equation (3), $u_{1}=t$ in this case, it follows that there is an equilibrium state of the population corresponding to each root of equation (79), and these equilibria are characterised by the gene ratios as follows:

$$
\left.\begin{array}{rll}
\text { I. } & u_{1}=0.5821, & u_{2}=0.3388 \\
\text { II. } & u_{1}=1 \cdot 0000, & u_{2}=1.0000 \\
\text { III. } & u_{1}=1.7179, & u_{2}=2.9512
\end{array}\right\}
$$

corresponding to the values given by Owen (1953).

The stability of each equilibrium can be tested by calculating the stability roots. These are obtained as the latent roots of the matrix $B=\left(b_{i j}\right)$ the elements of which are obtained from equations (67). Thus, for the equilibrium I, we find that

$$
B=\left(\begin{array}{ll}
0.3939 & 0.5206 \\
0.4057 & 0.6061
\end{array}\right)
$$

and hence the characteristic equation is

$$
\lambda^{2}-\lambda+0 \cdot 0275=0
$$

so that the stability roots are 0.9717 and $0 \cdot 0283$, and hence the equilibrium is stable. (Note that these values of the stability roots differ somewhat from those given by Owen.)

For equilibrium II we obtain

$$
B=\left(\begin{array}{cc}
\frac{1}{3} & \frac{1}{3} \\
\frac{43}{63} & \frac{43}{63}
\end{array}\right)
$$

and hence the characteristic equation is

$$
\lambda^{2}-\left(\frac{1}{3}+\frac{43}{63}\right) \lambda=0
$$

so that the stability roots are zero and $\frac{64}{63}=1 \cdot 0159$ agreeing with the values found by Owen and this equilibrium is unstable. 
Finally, for equilibrium III we obtain

$$
B=\left(\begin{array}{ll}
0.3939 & 0 \cdot 1764 \\
1 \cdot 1973 & 0 \cdot 6061
\end{array}\right)
$$

and hence the characteristic equation and the stability roots are identical with those for equilibrium I, which follows intuitively from the fact that in this example the system is invariant under the gene substitution $A \rightarrow a$. Owen's values for the stability roots of this equilibrium do not correspond.

In Owen's second example, the viability parameters are

$$
\left.\begin{array}{lll}
a_{1}=0.5, & h_{1}=1, & b_{1}=0.5 \\
a_{2}=4 \cdot 1, & h_{2}=1, & b_{2}=3.5
\end{array}\right\}
$$

so that

$$
\left.\begin{array}{l}
c_{1}=C_{1}=0 \\
c_{2}=6, \quad C_{2}=7 \cdot 2
\end{array}\right\}
$$

and thus the cubic equation (5) becomes

$$
f(t)=t^{3}-7 \cdot 2 t^{2}+6 t-1=0 .
$$

By inspection, equation (88) has a root close to $t=\frac{1}{\sqrt{2}}=0.7071$, and using Newton's method we can obtain a better approximation at $t=0 \cdot 7057$. The residual quadratic equation is then

$$
t^{2}-6 \cdot 4943 t+1 \cdot 4170=0
$$

with roots at $t=0.2261$ and $t=6.2682$. Hence there are three equilibria, characterised by the following gene ratios:

$$
\left.\begin{array}{rll}
\text { I. } & u_{1}=6.2682, & u_{2}=39.2903 \\
\text { II. } & u_{1}=0.7057, & u_{2}=0.4980 \\
\text { III. } & u_{1}=0.2261, & u_{2}=0.0511
\end{array}\right\}
$$

These values do not agree with those given by Owen (1953), due to the fact that Owen appears inadvertently to have interchanged the values of $\alpha$ and $\beta$. This in fact makes the transformation $A \rightarrow a$, which is thus the problem solved by Owen.

In order to examine the stability of the three equilibria, we note that for equilibrium I the $\left(b_{i j}\right)$ have the values

$$
B=\left(\begin{array}{ll}
0.7307 & 0.0215 \\
5.4010 & 0.2494
\end{array}\right)
$$

and the characteristic equation of $B$ is

$$
\lambda^{2}-0.9801 \lambda+0.0661=0
$$

with roots 0.90725 and 0.07285 so that this equilibrium is stable and the values of the stability roots agree with those given by Owen.

For equilibrium II, we obtain

$$
B=\left(\begin{array}{ll}
0.3595 & 0.4538 \\
0.5590 & 0.7666
\end{array}\right)
$$


and the characteristic equation

$$
\lambda^{2}-1 \cdot 1261 \lambda+0 \cdot 0219=0
$$

so that the stability roots are $1 \cdot 1063$ and 0.0198 , showing that this equilibrium is unstable, and differing, incidentally, from the values obtained by Owen.

Lastly, for equilibrium III the $\left(b_{i j}\right)$ have the values

$$
B=\left(\begin{array}{ll}
0 \cdot 6460 & 0 \cdot 7830 \\
0 \cdot 1879 & 0 \cdot 3850
\end{array}\right)
$$

and the characteristic equation of $B$ is

$$
\lambda^{2}-1 \cdot 0310 \lambda+0 \cdot 1016=0
$$

with roots 0.92065 and $0 \cdot 11035$ which agrees with the values found by Owen and the equilibrium is stable.

As a final remark, it is interesting to note that the stability roots for the equilibria of the system cited by $\mathrm{Li}$ (1967) can be obtained very quickly using equations (67). Li's example is characterised by the system of parameters

$$
\left.\begin{array}{lll}
a_{1}=7, & h_{1}=3, & b_{1}=7 \\
a_{2}=7, & h_{2}=15, & b_{2}=7
\end{array}\right\}
$$

and leads to a pair of symmetrically located stable equilibria given by the gene ratios

and

$$
\left.\begin{array}{ll}
u_{1}=\frac{1}{3}, & u_{2}=\frac{3}{5} \\
u_{1}=3, & u_{2}=\frac{5}{3}
\end{array}\right\}
$$

each of which is characterised by stability roots whose values are 0.9672 and 0.0328 ; together with an unstable equilibrium at $u_{1}=u_{2}=1$ with stability roots 1.018 and zero.

\section{Summary}

Owen's model of a genetical system in which the genotypic viabilities differ between sexes as well as between genotypes is discussed, and some of its properties are described.

Acknowledgment. I wish to thank Dr J.S. Gale for indicating a number of places in the manuscript where some clarification was necessary.

\section{RefERENCES}

BODMER, w. F. 1965. Differential fertility in population genetics models. Genetics, 51, 411-424.

BODMER, W. F., AND PARSONS, P. A. 1960. The initial progress of new genes with various genetic systems. Heredity, 15, 283-299.

EDWARDS, A. W. F. 1967. Fundamental theorem of natural selection. Nature, 215, 537-538.

Fisher, R. A. 1922. On the dominance ratio. Proc. Roy. Soc. Edinb., 42, 321-341.

FrobeniUs, G. 1912. Über Matrizen aus nicht negativen Elementen. Sitzungsber. Preuss. Akad. Wiss., Berlin, 456-477. 
HALDANE, J. B. s. 1924. A mathematical theory of natural and artificial selection. I. Trans. Camb. Phil. Soc., 23, 19-41.

HALDANE, J. B. s. 1926. A mathematical theory of natural and artificial selection III. Proc. Camb. Phil. Soc., 23, 363-372.

LI, c. c. 1963. Equilibrium under differential selection in the sexes. Evolution, 17, 493-496. LI, c. c. 1967. Genetic equilibrium under selection. Biometrics, 23, 397-484.

MANDEL, S. P. H. 1963. A note on the initial progress of new genes. Heredity, 18, 535-538. MANDEL, S. P. H. 1968. Fundamental theorem of natural selection. Nature, 220, 1251-1252. OWEN, A. R. G. 1952. A genetical system admitting of two stable equilibria. Nature, 170, 1127.

owEN, A. R. G. 1953. A genetical system admitting of two distinct stable equilibria under natural selection. Heredity, 7, 97-102.

PARSONs, P. A. 1961a. The initial progress of new genes with viability differences between sexes and with sex linkage. Heredity, 16, 103-107.

PARSONS, P. A. 1961b. The establishment of new genes in populations under various genetic systems. Proc. 2nd Int. Conference of Human Genetics, Rome.

PARSONS, P. A. 1962. The initial increase of a new gene under positive assortative mating. Heredity, 17, 267-276. 